the book, which is how to explain hierarchical group structures in traditional human societies, and the forms of indiscriminate altruism that often accompany them. (Richerson and Boyd seem to want to use the word 'always' here but, in a rare lapse of concentration, I think they seriously overstep the evidence.)

Conventional evolutionary explanations, such as kin selection and reciprocal altruism, work well for small-scale societies, like those found among monkeys and apes. But they do not work quite as well on the larger scale of human tribal societies, where kinship and interaction frequencies are too dilute to sustain indiscriminate altruism. When individuals' ecological and reproductive success depend on the success with which they can cooperatively solve the problems of survival, something is needed to prevent the age-old tension between selfand group-level interests tearing the fabric of groups apart. Something is needed to enforce commitment to the group, and that something, Richerson and Boyd argue, is culture, creating a sufficiently strong sense of group identity and conformity to enable groups to do their job for the individual.

In many ways, this book is really addressed to social scientists, who, as Richerson and Boyd remark, learned their evolutionary biology from the mischievous writings of Steven Jay Gould, but failed to realize that his seductive polemics have little empirical support. Functional explanations for natural phenomena are, they remind us, often difficult to see without a great deal of hard empirical work. Gouldian arguments of 'spandrels as non-adaptation' are, they remind us, usually at least as much 'just so stories' as adaptationist explanations - if not more so, as they are rarely backed by anything resembling empirical evidence. And it is evidence, they insist, that is the core to good evolutionary biology. No amount of armchair theorizing can substitute for hardearned natural-history knowledge, as the seminal examples of Aristotle (whose biology, incidentally, was outstanding, however bad his physics might have been) and Darwin remind us.

In many ways, this book is a plea to the social sciences to take seriously what evolutionary biology has to offer - to see it not as a threat intent on cannibalizing the socialscience niche, but rather as a source of useful techniques and conceptual devices that can be applied within a conventional socialsciences framework, asking conventional social-science questions. It is a book full of good sense and the kinds of intellectual rigour and clarity of writing that we have come to expect from the Boyd-Richerson stable.

Robin Dunbar is in the School of Biological Sciences, University of Liverpool, Crown Street, Liverpool L69 7ZB, UK.

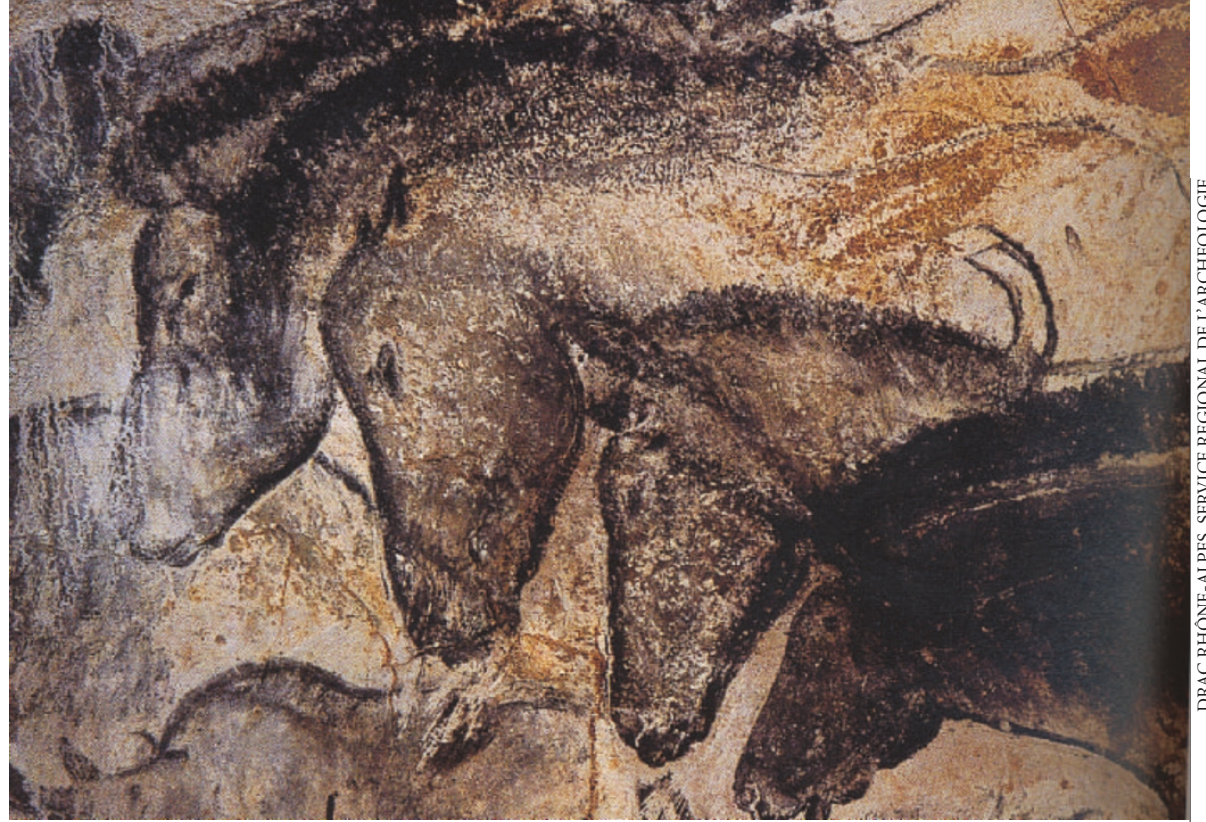

Quick on the draw? The age of paintings of horses at Chauvet cave in France is controversial.

\section{Charting the past}

\section{The Seventy Great Inventions}

of the Ancient World

edited by Brian M. Fagan

Thames \& Hudson: 2004. 304 pp.

$£ 24.95, \$ 40$

\section{Paul G. Bahn}

The 'Seventy Great' book franchise, which first appeared a few years ago, has until now seemed rather lightweight, focusing as it has on 'wonders' and 'mysteries'. The volumes were uniformly attractive and often well written, but they inevitably reminded one of those pointless 'top 10' and ' 100 best' shows that help to pad out the British TV schedules. It is likely that no two archaeologists would ever reach a consensus on which topics to include in such compilations.

But this new addition to the list is a distinct improvement: as a survey of ancient technology, it is far less random and certainly covers the basics. Edited by Brian Fagan, an eminent popularizer of archaeology, it presents almost all the major inventions of humankind, from its origins up to an undefined cut-off point. The 70 topics, we are told, were chosen from a potential list of hundreds, but the basis on which the selections were made, or who made them, is not revealed. Nevertheless, I think archaeologists would agree that few important topics have been omitted, and the team of 42 authors certainly includes a number of outstanding specialists.

Overall, the volume is very up-to-date and includes recent finds such as Germany's Nebra sky disc. Some of the essays are extremely good, notably those on pottery, watercraft and fermented beverages. Others are weaker, but this is an inevitable problem in such works - nobody can really be an expert on every period, and it shows.

In particular, some essays, such as those on clothing or jewellery, display a distinct lack of knowledge of the Stone Age evidence, despite the earliest periods being the most crucial to the book as a whole, as this is when these and many other inventions actually arose. For example, astonishingly, the essay on clothing makes no mention of Italy's Neolithic Iceman, who has made a huge contribution to our knowledge of early everyday wear, as opposed to burial garments; and the great Neolithic wooden well of Kückhoven, Germany, is absent from the essay on water supplies. The piece on boomerangs ignores not only those found in Tutankhamen's tomb, but also the great ivory specimen from Oblazowa, Poland, dating to at least 18,000 years ago; it may have been a ceremonial object, but nevertheless, trials with a facsimile proved it to be an efficient and aerodynamic weapon.

Among the topics that are missing completely, but which could well be argued to have been far more important to human life than board games, ball games, or codes and cyphers (all of which are included), one might mention levers and pulleys, ladders and scaffolds, steps and staircases, cordage and knots - and language. Other topics that are mentioned only briefly, such as mirrors and dentistry, should surely have been accorded much greater significance.

The book is also strangely inconsistent in its attitude to evidence. Some highly controversial items are accepted without a murmur, such as early dates for France's Chauvet cave, a late date for people reaching the Americas, and the Neanderthal 'flute' of Divje Babe, Slovenia, which is almost certainly natural, made by carnivores. Yet the early protofigurine of Berekhat Ram, Israel, is treated with great scepticism, despite having been the subject of two microscopic analyses.

But overall I can recommend this book as an entertaining pot-pourri. It presents a great deal of fascinating information in a highly readable and well illustrated way. Paul G. Bahn is a freelance archaeologist at 428 Anlaby Road, Hull HU3 6QP, UK. 